\title{
Investigations for genotoxic effects after exposure to crude 2,4,5-trichlorophenol
}

\author{
C E BLANK, ${ }^{2}$ PATRICIA COOKE, ${ }^{2}$ AND A M POTTER ${ }^{1}$ \\ From the Centre for Human Genetics, ${ }^{1}$ Sheffield, and the Cytogenetics Department, ${ }^{2}$ The City Hospital, \\ Nottingham, UK
}

ABSTRACT An investigation was designed to look for chromosome irregularity, as an indication of genotoxic effect, in individuals accidentally exposed to crude 2,4,5-trichlorophenol 10 years earlier. Dioxin was a trace contaminant. Measurement of chromosome aberration and sister chromatid exchange rate, both of which might be expected to be increased in individuals exposed to substantial quantities of chemical mutagenic or carcinogenic agents, or both, showed no significant deviation from that expected by chance.

In 1976 one of us (CEB) was asked by the medical adviser to Coalite Group Limited to look for evidence of genetic damage in individuals exposed to industrial pollution at the works of Coalite Oils and Chemicals Limited, Bolsover, England. The accident occurred in April 1968' during the production of 2,4,5-trichlorophenol (2,4,5-TCP) and led to the release of crude 2,4,5-TCP containing a higher than normal concentration of the impurity $2,3,7,8-$ tetrachlorodibenzodioxin (TCDD), commonly referred to as "dioxin."

An increase in chromosome aberration (figs 1 and 2) in lymphocyte culture is widely regarded as an indicator of exposure to mutagenic and carcinogenic agents. ${ }^{2}$ Measurement of chromosome aberration, and of sister chromatid exchange rate (see below), are the only practical methods for identifying and measuring genetic damage in individuals exposed to potential genotoxic agents. Chromosome aberration in lymphocyte culture may be observed many years after initial exposure. ${ }^{34}$ It was decided to compare the rate and type of aberration observed in "exposed" and "possibly exposed" individuals with that seen in a "control" (not believed to be exposed) series. Individuals were classified with respect to exposure by the medical adviser initiating the study, who also established a control series ( $G$ May, personal communication). Specimens of venous blood in heparin were obtained, coded, and delivered to the Centre for Human Genetics, Sheffield, over a period of six months from late 1977 to early 1978 . Examination for chromosome aberration took place

Received 28 October 1981 Accepted 18 January 1982 without knowledge of exposure history by the five cytogeneticists analysing the specimens.

A second method of investigation for detecting chromosome irregularity was used. Some specimens were also scored for sister chromatid exchanges (fig 3 ). This method is now recognised as the more sensitive indicator of genotoxic activity of chemical origin. ${ }^{5}$ Nevertheless, a change in sister chromatid exchange rate is not likely to persist long after exposure to a genotoxic agent has ceased.

In this report group $\mathrm{C}$ individuals were "known to be exposed" to dioxin pollution (with chloracne), group B had "possibly been exposed," and group. A were "controls."

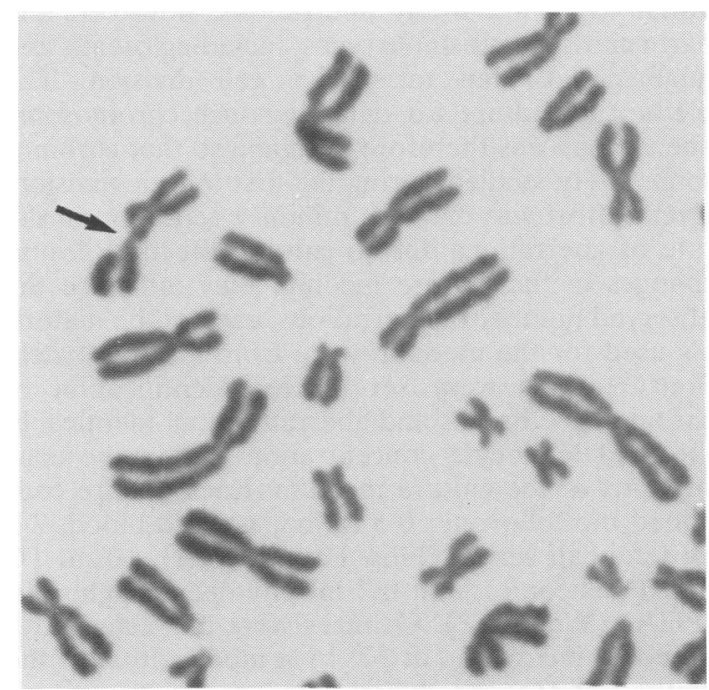

Fig 1 Chromosome breaks: arrowed. 


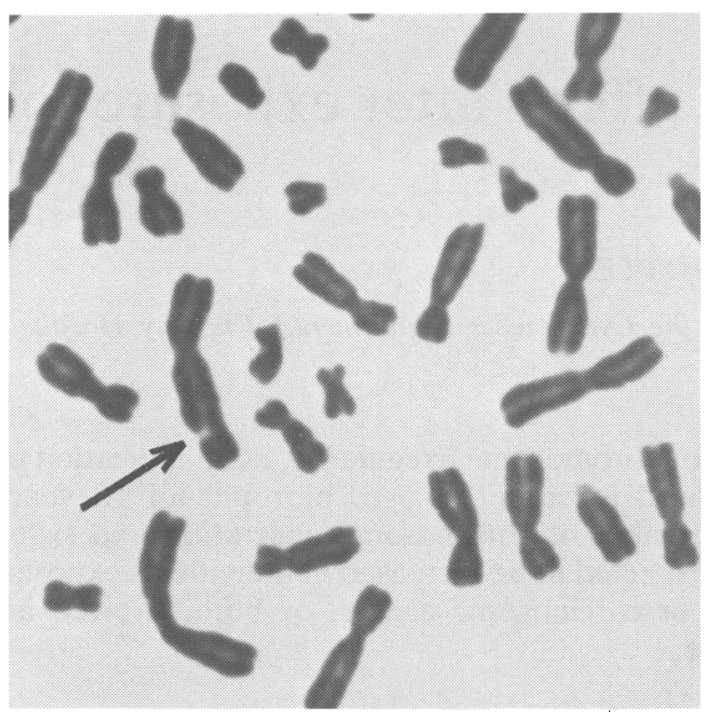

Fig 2 Chromatid break: arrowed.

\section{Material and methods}

Numbered blood samples were received for cytogenetic analysis over a period of six months. It was understood that specimens received in the same week would include samples from each of groups A, $\mathrm{B}$, and $\mathrm{C}$.

\section{LYMPHOCYTE CULTURE}

Bender and Gooch ${ }^{3}$ and Buckton et al ${ }^{4}$ described how radiation-induced chromosomal damage was observed in leucocyte culture 15-20 years after the initial exposure. Many aberrations, however, and particularly the unstable types, including breaks and quadriradials, fail to survive cell division. The method of culture for detecting such chromosome aberrations was therefore designed so that chromosomes were studied during the first mitotic division. Every effort was made to minimise variation in the rate of aberrations due to culture effects. Because changes in the culture medium may influence the observed number of aberrations, each of the materials used for the medium were from a single batch. Four cultures were set up for identification of chromosome breaks and aberrations, all identical in the final leukocyte concentration and in the constituents of the culture medium. Each culture contained the following: $0.5 \mathrm{ml}$ heparinised blood, 2.5 $\mathrm{ml}$ fetal calf serum (Flow Laboratories), $10 \mathrm{ml}$ TC 199 (Wellcome), and $0.2 \mathrm{ml}$ phytohaemagglutinin, (PHA) (Wellcome). Cultures were harvested after 48 hours incubation at $37^{\circ}$ by a modification of the method of Moorhead et al. ${ }^{6}$

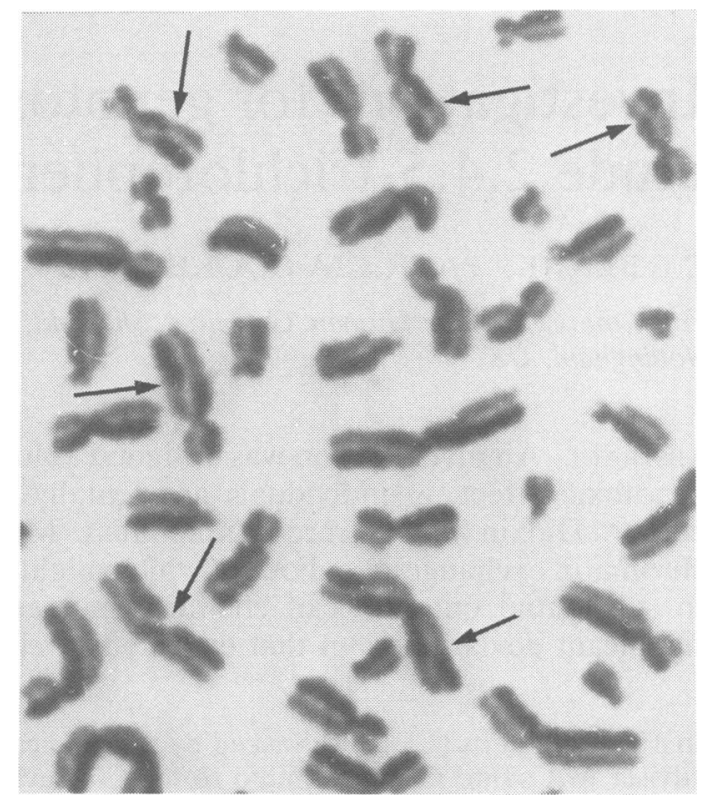

Fig 3 Sister chromatid exchanges: a few of them are arrowed.

Slides were prepared from all cultures, and these were stained with Leishman's stain (Gurr's Ltd). Fifty cells were then scored by one or other of five observers. These were taken from two separate cultures, and 25 cells were scored from each to check on any culture variation. The chromosome number was counted and the presence of gaps, breaks, or structural or numerical abnormalities scored. Breaks in either one chromatid (chromatid breaks) or two chromatids (chromosome breaks) were distinguished.

Gaps were defined as any discontinuity in the chromatid or chromosome greater than the width of a chromatid. Breaks were distinguished from gaps by having a displacement of the distal fragment from the remainder of the chromosome. Although structural abnormalities were noted, they were scored according to the number of breaks. Thus a quadriradial was scored as two chromatid breaks and a ring as two chromosome breaks. Finally, one cell was selected for full chromosome analysis in order to confirm a normal karyotype. Two individuals were found to have a congenital aneuploidy of the sex complement.

As the scoring was done by more than one observer, observer variation could obscure real intergroup differences. To measure this source of variation, a pool of five preparations was scored by each observer and "observer" variation statistically assessed (see below). 
Two further cultures were set up from each individual as previously described, except that these were incubated for 62 hours with $0.1 \mathrm{ml}$ 5-bromodeoxyuridine (BrDU). Because $\mathrm{BrDU}$ is photosensitive, cultures were protected against the light. Such a procedure ${ }^{7}$ enables the sister chromatid exchanges to be scored and is a sensitive method of assessing the breakage rate at the time of culture. These cultures were harvested by the same method as before but before staining were treated with Hoescht $33258\left(5 \times 10^{-6} \mathrm{~g} / \mathrm{ml}\right.$ final concentration) for 14 minutes and with two hours' exposure to ultraviolet light. They were then incubated in distilled water at $60^{\circ} \mathrm{C}$ for two hours and stained with Leishman's stain. A proportion of cells treated in this way shows distinct differences in the quality of staining of the two chromatids, and this allows scoring of an exchange that has taken place between chromatids. Ten cells were scored from each individual successfully banded in this way. Only cells of the very highest quality were used for scoring sister chromatid exchange rates.

Chromatid and chromosome gaps and breaks were scored in 124 individuals; 31 in group A, 55 in group B, and 38 in group C. Sister chromatid exchanges were scored in $\mathbf{4 0}$ individuals; eight from group A, 20 from group B, and 12 from group C.

\section{Results and statistical analysis*}

GAPS AND BREAKS

Since there were only minimal differences between the duplicate cultures of each individual these results were pooled - the data used therefore consist of the total number of chromatid or chromosome gaps and breaks present in $\mathbf{5 0}$ cells of an individual.

A distribution plot of the data showed an approximately Poisson distribution for chromatid and chromosome events. A computer program was used to analyse the data. This program (general linear interactive model (GLIM)) may be used to produce a $\chi^{2}$-based breakdown of sources of deviation in data of any specified distribution. The deviations produced by any particular factor may be assessed directly as a $\chi^{2}$. If the residual deviations are significant $(\mathrm{p}<1 \%)$, however, then the main factor items cannot be adequately assessed.

Preliminary analysis of the results obtained when all observers scored the same five individuals suggested that different observers may vary in the criteria on which they score events-particularly chromatid events. The main analysis was therefore

\footnotetext{
* Raw data may be obtained on application to Dr C E Blank, Centre for Human Genetics, 117 Manchester Road, Sheffield, England
}

broken down to analyse the difference between groups $\mathrm{A}, \mathrm{B}$, and $\mathrm{C}$ within observers.

Tables 1 and 2 show the results of the GLIM analyses for chromatid and chromosome events respectively, together with the mean number of such events per cell for each group.

Table 1 (chromatid events) has an extremely high residual item so that the main items cannot be adequately assessed. This high level of residual deviation may be due to the presence of some other factor or factors acting to produce variations between individuals that has not been taken into account. Such factors may include the age of the individual, the time at which the individual was studied, or the quality of the preparation obtained. An alternative, but perhaps less likely, hypothesis is that the underlying assumption of a Poisson distribution is incorrect.

Table 2 (chromosome events) has a small residual item that is acceptably low. This analysis shows no significant difference in the number of chromosome events between groups.

\section{TOTAL EVENTS (CHROMATID/CHROMOSOME GAPS AND BREAKS)}

A distribution plot of the frequency of all events showed few zero observations and less extreme skewness. Several transformations were therefore attempted on the data, and the transformation log $(X+1)$ was found to produce a reasonably symmetrical distribution. The analysis of variance technique is relatively resistant to minor deviations from normality, so this technique was used to analyse the transformed data.

Table 3 shows the results of this analysis with alternative breakdown of the "group" items taking into account the differences between observers, together with the mean number of total events per cell for each group. There was no significant difference between groups either overall or within observers.

\section{SISTER CHROMATID EXCHANGE}

A distribution plot of the data showed an approximately normal distribution. An analysis of variance technique was therefore applied. Table 4 shows the results of the analysis of variance together with the average number of sister chromatid exchanges per individual (in 10 cells) for each group.

More objective scoring is possible of these exchanges so observer variation should not be a problem. As a check, the variation between groups was compared with the within-group (between individuals) variation for each observer separately.

The analyses showed no significant variation in the frequency of sister chromatid exchange between groups, either overall or within observers. 
Table 1 Chromatid events

\begin{tabular}{|c|c|c|c|}
\hline $\begin{array}{l}\text { (a) GLIM analysis } \\
\text { Component }\end{array}$ & 2 & $d f$ & $p$ \\
\hline $\begin{array}{l}\text { Between observers } \\
\text { Groups within observers } \\
\text { Residual deviation }\end{array}$ & $\begin{array}{r}69 \cdot 6 \\
19 \cdot 7 \\
206 \cdot 1\end{array}$ & $\begin{array}{r}4 \\
10 \\
109\end{array}$ & $<0.1 \%$ \\
\hline Total & 295.4 & 123 & \\
\hline \multicolumn{3}{|c|}{$\begin{array}{r}\text { (b) Mean number of chromatid events per cell } \\
\text { Group } A \quad \text { Group } B\end{array}$} & Group $C$ \\
\hline $\begin{array}{l}\text { No of cells } \\
\text { Mean }\end{array}$ & & 556 & $\begin{array}{l}1900 \\
0.0600\end{array}$ \\
\hline
\end{tabular}

Table 2 Chromosome events

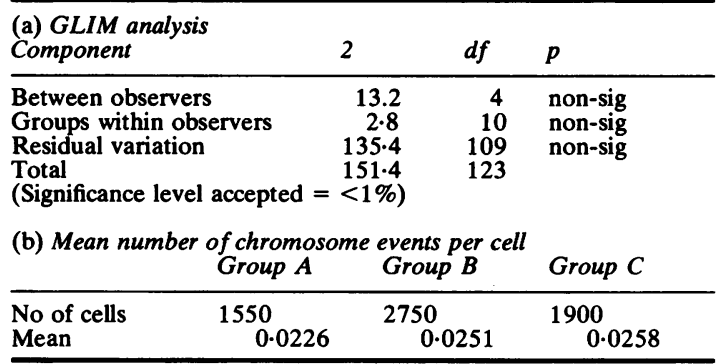

Table 3 Total events: chromatid/chromosome gaps and breaks

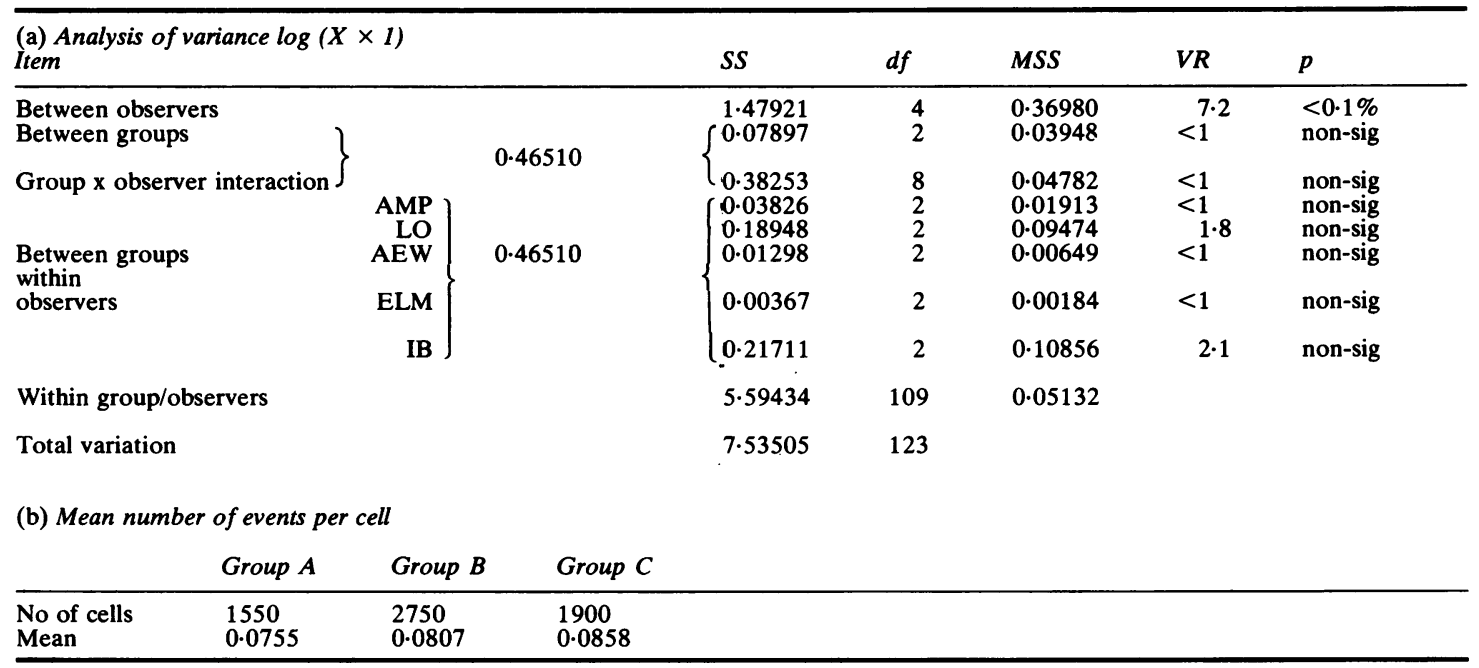

Table 4 Sister chromatid exchange

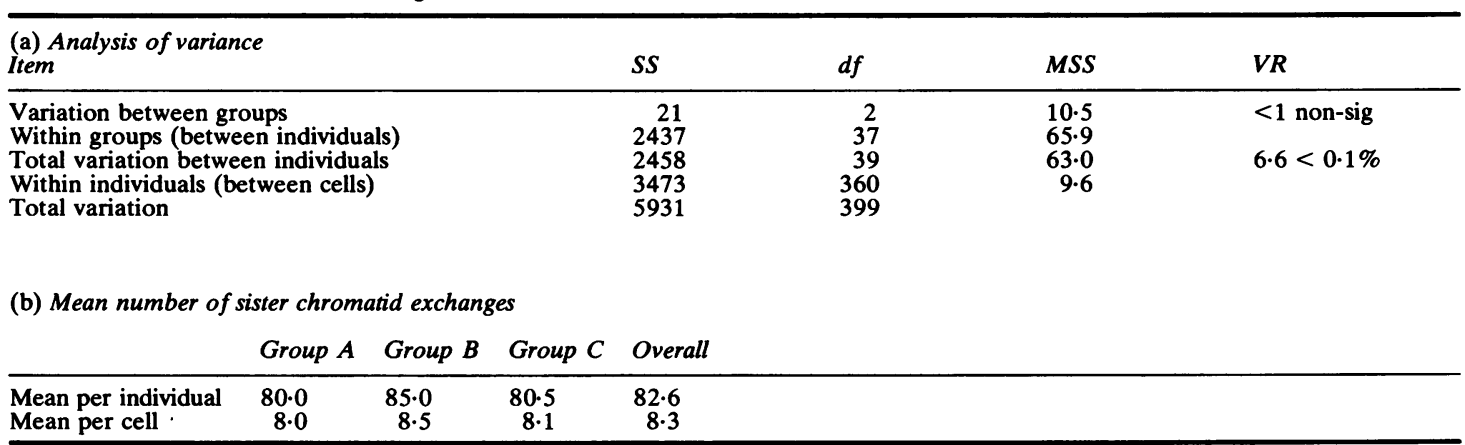




\section{Discussion}

The statistical methods used are such that significant differences when comparing $A$ and $B$ against $C$, or any other combination of $\mathrm{A}, \mathrm{B}$, and $\mathrm{C}$, would have been apparent. With respect to chromatid/ chromosome gaps and breaks there is no statistically significant variation between groups (table 3); although it is of interest that the mean score per cell for chromatid/chromosome irregularity is highest in group C (known to be exposed) and lowest in group A (controls). Again, no significant variation in frequency of sister chromatid exchanges between groups (table 4) is observed.

In conclusion, it was evident that measurement of chromosome aberration, or mitotic events (sister chromatid exchange rate), which might be expected to be increased in individuals exposed to substantial quantities of chemical mutagenic/carcinogenic agents showed no statistically significant differences between the groups studied.

We are grateful to various members of the staff of the Centre for Human Genetics, Sheffield, for undertaking the cytogenetic analysis and to the computer services department of the University of Nottingham for running the GLIM analysis.

\section{References}

' May G. Chloracne from the accidental production of tetrachlorodibenzodioxin. $\mathrm{Br} J$ Ind Med 1973;30:276-83.

${ }^{2}$ Evans HJ. Environmental agents and chromosome damage in somatic cells of man: in vivo studies. In: Boyce AJ, ed. Chromosome variations in human evolution. London: Taylor and Francis, 1975:63-82.

${ }^{3}$ Bender MA, Gooch PC. Persistent chromosome aberrations in irradiated human subjects. Radiat Res 1962;16:44-53.

4 Buckton KE, Jacobs PA, Court Brown WM, Doll R. A study of the chromosome damage persisting after $x$-ray therapy for ankylosing spondylitis. Lancet 1962;ii:676-82.

5 Evans HG, Lloyd DC, eds. Mutagen-induced chromosome damage in man. Edinburgh: University Press, (in press).

- Moorhead PS, Nowell PC, Mellman WJ, Battips DM, Hungerford DA. Chromosome preparations of leukocytes cultured from human peripheral blood. Exp Cell Res 1960;20:613-6.

7 Perry P, Wolff, S. New giemsa method for the differential staining of sister chromatids. Nature 1974:251:156-8. 